\title{
Reply to: Wanted: Automated objective proficiency assessment metrics for the FAST exam (and other POCUS studies)
}

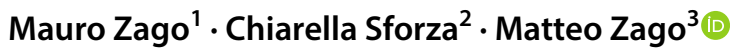

Received: 30 May 2019 / Accepted: 4 June 2019 / Published online: 12 June 2019

(c) Springer-Verlag GmbH Germany, part of Springer Nature 2019

We thank Drs. Bell and Holden for their appreciation and valuable insights [1] about our recent work on the evaluation of sonographers' skills by means of hand motion analysis [2].

We perfectly agree with them that the measurement of probe motion should be coupled to that of hand motion. Unfortunately, in our experimental set, the probe was almost always occluded, thus it could not be recorded by the cameras.

This is one limitation of optoelectronic devices, as already discussed in our investigation [2]. In contrast, Bell et al. [3] used a different technology (electromagnetic tracking) that is free from this limitation but that can measure only a reduced number of markers.

To overcome this problem, we calculated both conventional and standardized metrics to assess hand motion, and found useful information related to experience (both groups performed almost the same number of movements per second, but experts quickly performed more probe heading adjustments in a reduced working volume) that are currently being used for didactic purposes. All this information can partially compensate for the current lack of a direct assessment of the action at the ending edge of the tool.

In practical terms, thanks to the results of our research, we are now limiting the elbow and forearm excursion during US training. This enhances the trainee perception of the more efficient (time and path length) and effective (achievement

Matteo Zago

matteo2.zago@polimi.it

1 Department of Surgery, Minimally Invasive Surgery Unit, Policlinico San Pietro, Via Forlanini 15, Ponte San Pietro, 24036 Bergamo, Italy

2 Department of Biomedical Sciences for Health, Università degli Studi di Milano, via Mangiagalli 31, 20133 Milano, Italy

3 Dipartimento di Elettronica, Informazione e Bioingegneria, Politecnico di Milano, via Golgi 39, 20133 Milano, Italy of the proper views) handling of the instrument, speeding up the improvements of the wrist motion, and the ability to tilting and fanning the probe (the action end of the probe reported by Holden [4]).

We are grateful to Drs. Bell and Holden for underlining this important part of our common research. We are already working toward the definition of a new protocol that may overcome the current limitations.

\section{Compliance with ethical standards}

Conflict of interest Chiarella Sforza has no conflict of interest to declare. Mauro Zago has no conflict of interest to declare. Matteo Zago has no conflict of interest to declare.

\section{References}

1. Bell CR, Holden MS. Wanted: automated objective proficiency assessment metrics for the FAST exam (and other POCUS studies). Eur J Trauma Emerg Surg. 2019. https://doi.org/10.1007/ s00068-019-01156-8.

2. Zago M, Sforza C, Mariani D, Marconi M, Biloslavo A, La Greca A, Kurihara H, Casamassima A, Bozzo S, Caputo F, Galli M, Zago M. Educational impact of hand motion analysis in the evaluation of fast examination skills. Eur J Trauma Emerg Surg. 2019. https://doi.org/10.1007/s00068-019-01112-6.

3. Bell CR, McKaigney CJ, Holden M, Fichtinger G, Rang L. Sonographic accuracy as a novel tool for point-of-care ultrasound competency assessment. AEM Educ Train. 2017;1(4):316-24.

4. Holden MS, Keri Z, Ungi T, Fichtinger G. Overall proficiency assessment in point-of-care ultrasound interventions: the stopwatch is not enough. In: Cardoso $\mathrm{M}$, et al., editors. Imaging for patient-customized simulations and systems for point-of-care ultrasound, international workshops proceedings. Berlin: Cham; 2017. p. 146-153. 